\title{
THE CP-MATRIX APPROXIMATION PROBLEM
}

\author{
JINYAN FAN AND ANWA ZHOU
}

\begin{abstract}
A symmetric matrix $A$ is completely positive (CP) if there exists an entrywise nonnegative matrix $V$ such that $A=V V^{T}$. In this paper, we study the CP-matrix approximation problem of projecting a matrix onto the intersection of a set of linear constraints and the cone of $\mathrm{CP}$ matrices. We formulate the problem as the linear optimization with the norm cone and the cone of moments. A semidefinite algorithm is presented for the problem. A $\mathrm{CP}$-decomposition of the projection matrix can also be obtained if the problem is feasible.
\end{abstract}

\section{INTRODUCTION}

A real $n \times n$ symmetric matrix $A$ is completely positive (CP) if there exist nonnegative vectors $v_{1}, \cdots, v_{r} \in \mathbb{R}_{+}^{n}$ such that

$$
A=v_{1} v_{1}^{T}+\cdots+v_{r} v_{r}^{T},
$$

where $r$ is called the length of the decomposition (1.1). The smallest $r$ in the above is called the CP-rank of $A$. If $A$ is CP, we call (1.1) a CP-decomposition of $A$. So, $A$ is $\mathrm{CP}$ if and only if $A=V V^{T}$ for an entrywise nonnegative $V$. Clearly, a CP-matrix is double nonnegative, i.e., it is not only positive semidefinite but also nonnegative entrywise.

Let $\mathcal{S}_{n}$ be the set of real $n \times n$ symmetric matrices. For a cone $\mathcal{C} \subseteq \mathcal{S}_{n}$, the dual cone of $\mathcal{C}$ is defined as

$$
\mathcal{C}^{*}:=\left\{B \in \mathcal{S}_{n}: A \bullet B \geq 0 \text { for all } A \in \mathcal{C}\right\},
$$

where $A \bullet B:=\operatorname{trace}\left(A^{T} B\right)$ is the standard inner product on $\mathbb{R}^{n \times n}$. Denote

$$
\begin{aligned}
& \mathcal{C P}_{n}=\left\{A \in \mathcal{S}_{n}: A=V V^{T} \text { with } V \geq 0\right\}, \text { the completely positive cone, } \\
& \mathcal{C O} \mathcal{P}_{n}=\left\{B \in \mathcal{S}_{n}: x^{T} B x \geq 0 \text { for all } x \geq 0\right\}, \text { the copositive cone. }
\end{aligned}
$$

Both $\mathcal{C P}_{n}$ and $\mathcal{C O P}{ }_{n}$ are proper cones (i.e. closed, pointed, convex and fulldimensional). Moreover, they are dual to each other [17. A variety of NP-hard problems can be formulated as optimization problems over the completely positive cone or the copositive cone. Interested readers are referred to [2, 6, 7, 8, 4, 12, 15] for the work in the field.

The important applications of the CP cone motivate people to study whether a matrix is $\mathrm{CP}$ or not. However, checking the membership in $\mathcal{C P}$. has been shown NPhard, while checking the membership in $\mathcal{C O P}{ }_{n}$ co-NP-hard [13, 26]. It is generally difficult to treat $\mathcal{C P}$ (or $\mathcal{C O P}$ ) directly. A standard approach is to approximate

2000 Mathematics Subject Classification. Primary: 90C20, 90C22, 90C26.

Key words and phrases. completely positive matrices, CP projection, CP-matrix approximation, linear optimization with moments, semidefinite algorithm.

The first author is partially supported by NSFC 11171217 . 
it by simpler and more tractable cones [12, 14, 3, 32, 24. By Nie's approach proposed in 27, 28, Zhou and Fan [36] presented a semidefinite algorithm for the $\mathrm{CP}$-matrix completion problem, which includes the $\mathrm{CP}$ checking as a special case; a CP-decomposition for a general CP-matrix can also be found by the algorithm. The approach is also applied to check interiors of the completely positive cone 37.

In [34, Sponseldur and Dür considered the problem of projecting a matrix onto the cones of copositive and completely positive matrices. Unlike projecting onto the cones of nonnegative matrices and positive semidefinite matrices, projecting onto either $\mathcal{C P}_{n}$ or $\mathcal{C O P}{ }_{n}$ is a nontrivial task in view of the NP-complexity results [13, 26]. Sponseldur and Dür used polyhedral approximations of $\mathcal{C O} \mathcal{P}_{n}$ to compute the projection of a matrix onto $\mathcal{C O P}{ }_{n}$ and the projection onto $\mathcal{C P}$ by a dual approach.

In this paper, we consider the general $\mathrm{CP}$-matrix approximation problem stated as:

$$
\begin{array}{cl}
\min _{X} & \|X-C\|_{p} \\
\text { s.t. } & A_{i} \bullet X=b_{i}, i=1, \ldots, m_{e}, \\
& A_{i} \bullet X \geq b_{i}, i=m_{e}+1, \ldots, m, \\
& X \in \mathcal{C P} \mathcal{P}_{n},
\end{array}
$$

where $C, A_{i} \in \mathcal{S}_{n}, b_{i} \in \mathbb{R}(i=1, \ldots, m)$, and $\|\cdot\|_{p}$ is the $p$-norm $(p=1,2, \infty$ or $F)$. The problem is projecting a symmetric matrix onto the intersection of a set of linear constraints and the complete positive cone.

Specially, if $C=0$, then (1.2) becomes the feasibility problem of finding a matrix in the intersection of a set of linear constraints and the CP cone, which has the minimum $p$-norm.

If there are no linear constraints, (1.2) is reduced to the $\mathrm{CP}$ projection problem

$$
\begin{array}{cl}
\min _{X} & \|X-C\|_{p} \\
\text { s.t. } & X \in \mathcal{C} \mathcal{P}_{n} .
\end{array}
$$

Hence, the CP projection problem is a special case of (1.2). Clearly, (1.3) is always feasible and has a solution. If the minimum is zero, then the projection matrix of $C$ onto $\mathcal{C P}_{n}$ is itself, which implies that $C$ is CP. If the minimum is nonzero, then $C$ is not $\mathrm{CP}$. So, solving the $\mathrm{CP}$ projection problem (1.3) provides a way to check whether $C$ is CP.

In this paper, we formulate (1.2) as a linear optimization problem with the cone of moments and the $p$-norm cone, then propose a semidefinite algorithm for it. If (1.2) is infeasible, we can get a certificate for it. If (1.2) is feasible, we can get a projection matrix of $C$ onto the set of linear constraints and the $\mathrm{CP}$ cone. Moreover, a CP-decomposition of the projection matrix can also be obtained.

The paper is organized as follows. In section 2, we review the norm cone and its dual cone, and characterize the CP matrix as a moment sequence. In section 3 , we show how to formulate (1.2) as a linear optimization problem with the norm cone and the cone of moments; its dual problem is also given. We present a smidefinite algorithm for (1.2) and study its convergence properties in section 4 . Some computational results are given in section 5. Finally, we conclude the paper in section 6. 


\section{Preliminaries}

In this section, we first give the dual norm of the $p$-norm on $\mathcal{S}_{n}$ for $p=1,2, \infty$ and $F$ respectively; the $p$-norm cone and its dual cone are also given. Then we characterize $\mathrm{CP}$ matrices as moments, and review some basics about moments and localizing matrices, as well as the semidefinite relaxations of the $\mathrm{CP}$ cone (cf. [21, 22, 25, 23, 27]).

2.1. $p$-norm cone and its dual. For $A \in \mathbb{R}^{n \times n}$, the $p$-norms of $A(p=1,2, \infty, F)$ are defined by:

$$
\begin{aligned}
& \|A\|_{1}=\max _{j} \sum_{i=1}^{n}\left|A_{i j}\right|, \quad \text { the maximum absolute column sum norm or 1-norm, } \\
& \|A\|_{2}=\left(\lambda_{\max }\left(A^{T} A\right)\right)^{1 / 2}, \quad \text { the spectral norm or 2-norm, } \\
& \|A\|_{\infty}=\max _{i} \sum_{j=1}^{n}\left|A_{i j}\right|, \quad \text { the maximum absolute row sum norm or } \infty \text {-norm, } \\
& \|A\|_{F}=\left(\operatorname{trace}\left(A^{T} A\right)\right)^{1 / 2}, \quad \text { the Frobenius norm or } F \text {-norm. }
\end{aligned}
$$

Note that, when $A \in \mathcal{S}_{n}$, the 1-norm is the same as $\infty$-norm.

Let $\|\cdot\|$ be a norm on $\mathcal{S}_{n}$. The associated dual norm, denoted by $\|\cdot\|_{*}$, is defined by

$$
\|A\|_{*}=\sup \{A \bullet X:\|X\| \leq 1\},
$$

(cf. [5, Section A.1.6]). It can be proved that the dual norm of the $p$-norm for $p=1,2, \infty$ and $F$ are:

$$
\begin{aligned}
\|A\|_{1 *} & =\sum_{j=1}^{n} \max _{i}\left|A_{i j}\right| \\
\|A\|_{2 *} & =\operatorname{trace}\left(\left(A^{T} A\right)^{1 / 2}\right), \\
\|A\|_{\infty *} & =\sum_{i=1}^{n} \max _{j}\left|A_{i j}\right| \\
\|A\|_{F *} & =\|A\|_{F}
\end{aligned}
$$

For $\|\cdot\|$ on $\mathcal{S}_{n}$, the norm cone is defined by

$$
\mathcal{K}=\left\{(X, s) \in \mathcal{S}_{n} \times \mathbb{R}_{+}:\|X\| \leq s\right\},
$$

where $\mathcal{S}_{n} \times \mathbb{R}_{+}$is the Cartesian product of $\mathcal{S}_{n}$ and $\mathbb{R}_{+}$. The dual cone of $\mathcal{K}$ is defined by

$$
\mathcal{K}^{*}=\left\{(Y, t) \in \mathcal{S}_{n} \times \mathbb{R}_{+}: X \bullet Y+s t \geq 0 \text { for all }(X, s) \in \mathcal{S}_{n} \times \mathbb{R}_{+}\right\} .
$$

For the $p$-norm cone $(p=1,2, \infty$ and $F)$,

$$
\mathcal{K}_{p}=\left\{(X, s) \in \mathcal{S}_{n} \times \mathbb{R}_{+}:\|X\|_{p} \leq s\right\},
$$

we can prove that the dual cone of $\mathcal{K}_{p}$ is

$$
\mathcal{K}_{p}^{*}=\left\{(Y, t) \in \mathcal{S}_{n} \times \mathbb{R}_{+}:\|Y\|_{p *} \leq t\right\} .
$$


2.2. Characterization as moments. A symmetric matrix $A \in \mathcal{S}_{n}$ can be identified by a vector consisting of its upper triangular entries, i.e.

$$
\operatorname{vech}(A)=\left(A_{11}, A_{12}, \ldots, A_{1 n}, A_{22}, \ldots, A_{2 n}, A_{33}, \ldots, A_{n n}\right)^{T} .
$$

Let $\mathbb{N}$ be the set of nonnegative integers. For $\alpha=\left(\alpha_{1}, \cdots, \alpha_{n}\right) \in \mathbb{N}^{n}$, denote $|\alpha|:=\alpha_{1}+\cdots+\alpha_{n}$. Let

$$
E:=\left\{\alpha \in \mathbb{N}^{n}:|\alpha|=2\right\} .
$$

Then, $A$ can also be identified as

$$
a=\left(a_{\alpha}\right)_{\alpha \in E} \in \mathbb{R}^{E}, \quad a_{\alpha}=A_{i j} \text { if } \alpha=e_{i}+e_{j}, i \leq j,
$$

where $e_{i}$ is the $i$-th unit vector in $\mathbb{R}^{n}$ and $\mathbb{R}^{E}$ denotes the space of real vectors indexed by $\alpha \in E$. We call $a$ an $E$-truncated moment sequence ( $E$-tms).

Let

$$
\Delta=\left\{x \in \mathbb{R}^{n}: x_{1}^{2}+\cdots+x_{n}^{2}-1=0, x_{1} \geq 0, \cdots, x_{n} \geq 0\right\}
$$

be the nonnegative part of the unit sphere. Every nonnegative vector is a multiple of a vector in $\Delta$. So, by (1.1), $A \in \mathcal{C P}_{n}$ if and only if there exist $\rho_{1}, \cdots, \rho_{r}>0$ and $u_{1}, \cdots, u_{r} \in \Delta$ such that

$$
A=\rho_{1} u_{1} u_{1}^{T}+\cdots+\rho_{l} u_{r} u_{r}^{T} .
$$

The $E$-truncated $\Delta$-moment problem $(E$-T $\Delta \mathrm{MP})$ studies whether or not a given $E$-tms $a$ admits a $\Delta$-measure $\mu$, i.e., a nonnegative Borel measure $\mu$ supported in $\Delta$ such that

$$
a_{\alpha}=\int_{\Delta} x^{\alpha} d \mu, \quad \forall \alpha \in E,
$$

where $x^{\alpha}:=x_{1}^{\alpha_{1}} \cdots x_{n}^{\alpha_{n}}$. A measure $\mu$ satisfying the above is called a $\Delta$-representing measure for $a$. A measure is called finitely atomic if its support is a finite set, and is called $r$-atomic if its support consists of at most $r$ distinct points.

Hence, by (2.4), a symmetric matrix $A$, with the identifying vector $a \in \mathbb{R}^{E}$, is completely positive if and only if $a$ admits an $r$-atomic $\Delta$-measure, i.e.,

$$
a=\rho_{1}\left[u_{1}\right]_{E}+\cdots+\rho_{r}\left[u_{r}\right]_{E},
$$

where each $\rho_{i}>0, u_{i} \in \Delta$ and

$$
\left[u_{i}\right]_{E}:=\left(u_{i}^{\alpha}\right)_{\alpha \in E}, \quad i=1, \ldots, r .
$$

Denote

$$
\mathcal{R}=\left\{a \in \mathbb{R}^{E}: a \text { admits a } \Delta \text {-measure }\right\} .
$$

Then, $\mathcal{R}$ is the CP cone (cf. 28]). Hence,

$$
A \in \mathcal{C} \mathcal{P}_{n} \quad \text { if and only if } \quad a \in \mathcal{R} .
$$


2.3. Localizing matrices and flatness. Let $E$ and $\Delta$ be given in (2.2) and (2.3), respectively. Denote

$$
\mathbb{R}[x]_{E}:=\operatorname{span}\left\{x^{\alpha}: \alpha \in E\right\} .
$$

We say $\mathbb{R}[x]_{E}$ is $\Delta$-full if there exists a polynomial $p \in \mathbb{R}[x]_{E}$ such that $\left.p\right|_{\Delta}>0$ (i.e. $p(u)>0$ for all $u \in \Delta$ ). It is shown in [?] that the dual cone of $\mathcal{R}$ is

$$
\mathcal{P}=\left\{p \in \mathbb{R}[x]_{E}: p(x) \geq 0, \forall x \in \Delta\right\} .
$$

An $E$-tms $a \in \mathbb{R}^{E}$ defines a Riesz functional $F_{a}$ acting on $\mathbb{R}[x]_{E}$ as

$$
F_{a}\left(\sum_{\alpha \in E} p_{\alpha} x^{\alpha}\right):=\sum_{\alpha \in E} p_{\alpha} a_{\alpha}
$$

For convenience, we also denote the inner product $\langle p, a\rangle:=F_{a}(p)$.

Let

$$
\mathbb{N}_{d}^{n}:=\left\{\alpha \in \mathbb{N}^{n}:|\alpha| \leq d\right\} \quad \text { and } \quad \mathbb{R}[x]_{d}:=\operatorname{span}\left\{x^{\alpha}: \alpha \in \mathbb{N}_{d}^{n}\right\} .
$$

For $s \in \mathbb{R}^{\mathbb{N}_{2 k}^{n}}$ and $q \in \mathbb{R}[x]_{2 k}$, the $k$-th localizing matrix of $q$ generated by $s$ is the symmetric matrix $L_{q}^{(k)}(s)$ satisfying

$$
F_{s}\left(q p^{2}\right)=\operatorname{vec}(p)^{T}\left(L_{q}^{(k)}(s)\right) \operatorname{vec}(p), \quad \forall p \in \mathbb{R}[x]_{k-\lceil\operatorname{deg}(q) / 2\rceil} .
$$

In the above, $\operatorname{vec}(p)$ denotes the coefficient vector of polynomial $p$ in the graded lexicographical ordering, and $\lceil t\rceil$ denotes the smallest integer that is not smaller than $t$. In particular, when $q=1, L_{1}^{(k)}(s)$ is called a $k$-th order moment matrix and denoted as $M_{k}(s)$. We refer to [27, 18, 16] for more details about localizing and moment matrices.

Denote the polynomials:

$$
h(x):=x_{1}^{2}+\cdots+x_{n}^{2}-1, g_{0}(x):=1, g_{1}(x):=x_{1}, \cdots, g_{n}(x):=x_{n} .
$$

Note that $\Delta$ given in (2.3) is nonempty compact. It can also be described equivalently as

$$
\Delta=\left\{x \in \mathbb{R}^{n}: h(x)=0, g(x) \geq 0\right\},
$$

where $g(x)=\left(g_{0}(x), g_{1}(x), \cdots, g_{n}(x)\right)$. As shown in [27, a necessary condition for $s \in \mathbb{R}^{\mathbb{N}_{2 k}^{n}}$ to admit a $\Delta$-measure is

$$
L_{h}^{(k)}(s)=0 \quad \text { and } \quad L_{g_{j}}^{(k)}(s) \succeq 0, \quad j=0,1, \cdots, n,
$$

where $L_{g_{j}}^{(k)}(s) \succeq 0$ means $L_{g_{j}}^{(k)}(s)$ is symmetric positive semidefinite. If, in addition to (2.12), $s$ satisfies the rank condition

$$
\operatorname{rank} M_{k-1}(s)=\operatorname{rank} M_{k}(s),
$$

then $s$ admits a unique $\Delta$-measure, which is $\operatorname{rank} M_{k}(s)$-atomic (cf. Curto and Fialkow [11). We say $s$ is flat if both (2.12) and (2.13) are satisfied.

Given two tms' $\bar{a} \in \mathbb{R}^{\mathbb{N}_{d}^{n}}$ and $\overline{\bar{a}} \in \mathbb{R}^{\mathbb{N}_{e}^{n}}$, we say $\overline{\bar{a}}$ is an extension of $\bar{a}$, if $d \leq e$ and $\bar{a}_{\alpha}=\overline{\bar{a}}_{\alpha}$ for all $\alpha \in \mathbb{N}_{d}^{n}$. If $\overline{\bar{a}}$ is flat and extends $\bar{a}$, we say $\overline{\bar{a}}$ is a flat extension of $\bar{a}$. We denote by $\left.\overline{\bar{a}}\right|_{E}$ the subvector of $\overline{\bar{a}}$, whose entries are indexed by $\alpha \in E$. Note that an $E$-tms $a \in \mathbb{R}^{E}$ admits a $\Delta$-measure if and only if it is extendable to a flat tms $\tilde{a} \in \mathbb{R}^{\mathbb{N}_{2 k}^{n}}$ for some $k$ (cf. [27]). By (2.7), we have that

$$
A \in \mathcal{C P} \mathcal{P}_{n} \text { if and only if } a \text { has a flat extension. }
$$


2.4. Semidefinite relaxations. We call a subset $I \subseteq \mathbb{R}[x]$ an ideal if $I+I \subseteq I$ and $I \cdot \mathbb{R}[x] \subseteq I$. For a tuple $\chi=\left(\chi_{1}, \ldots, \chi_{m}\right)$ of polynomials in $\mathbb{R}[x]$, denote by $I(\chi)$ the ideal generated by $\chi_{1}, \ldots, \chi_{m}$. The smallest ideal containing all $\chi_{i}$ is the set $\chi_{1} \mathbb{R}[x]+\cdots+\chi_{m} \mathbb{R}[x]$. A polynomial $f \in \mathbb{R}[x]$ is called a sum of squares (SOS) if there exist $f_{1}, \ldots, f_{k} \in \mathbb{R}[x]$ such that $f=f_{1}^{2}+\cdots+f_{k}^{2}$.

Let $h$ and $g$ be as in (2.11). Denote

$$
I_{2 k}(h)=\{h(x) \phi(x): \operatorname{deg}(h \phi) \leq 2 k\},
$$

and

$$
Q_{k}(g)=\left\{\sum_{j=0}^{n} g_{j} \varphi_{j}: \text { each } \operatorname{deg}\left(g_{j} \varphi_{j}\right) \leq 2 k \text { and } \varphi_{j} \text { is } \operatorname{SOS}\right\} .
$$

Then, $I(h)=\bigcup_{k \in \mathbb{N}} I_{2 k}(h)$ is the ideal generated by $h$, and $Q(g)=\bigcup_{k \in \mathbb{N}} Q_{k}(g)$ is the quadratic module generated by $g$ (cf. [28). We say $I(h)+Q(g)$ is archimedean if there exists $R>0$ such that $R-\|x\|^{2} \in I(h)+Q(g)$. Clearly, if $f \in I(h)+Q(g)$, then $\left.f\right|_{\Delta} \geq 0$. Conversely, if $\left.f\right|_{\Delta}>0$ and $I(h)+Q(g)$ is archimedean, then $f \in I(h)+Q(g)$. This is due to Putinars Positivstellensatz (cf. [33]).

For each $k \in \mathbb{N}$, denote

$$
\Psi_{k}=\left\{p \in \mathbb{R}[x]_{E}: p \in I_{2 k}(h)+Q_{k}(g)\right\} .
$$

Note that $E$ is finite, $\mathbb{R}[x]_{E}$ is $\Delta$-full because $p=\left.\sum_{i=1}^{n} x_{i}^{2}\right|_{\Delta}>0$, and $I(h)+Q(g)$ is archimedean because $1-\|x\|^{2}=-h(x) \in I(h)+Q(g)$. By [28, Propositions 3.5], we have

$$
\Psi_{1} \subseteq \cdots \subseteq \Psi_{k} \subseteq \Psi_{k+1} \subseteq \cdots \subseteq \mathcal{P}
$$

Moreover,

$$
\operatorname{int}(P) \subseteq \bigcup_{k} \Psi_{k} \subseteq P .
$$

Correspondingly, for each $k \in \mathbb{N}$, denote

$$
\Gamma_{k}=\left\{s \in \mathbb{R}^{\mathbb{N}_{2 k}^{n}}: L_{h}^{(k)}(s)=0, L_{g_{j}}^{(k)}(s) \succeq 0, j=0,1, \cdots, n\right\},
$$

and

$$
\Upsilon_{k}=\left\{\left.s\right|_{E}: s \in \Gamma_{k}\right\},
$$

(If $k<\operatorname{deg}(E) / 2, \Upsilon_{k}$ is defined to be $\mathbb{R}^{E}$, by default). Since $E$ is finite, $\mathbb{R}[x]_{E}$ is $\Delta$-full and $I(h)+Q(g)$ is archimedean, by [28, Proposition 3.3], we have

$$
\Upsilon_{1} \supseteq \cdots \supseteq \Upsilon_{k} \supseteq \Upsilon_{k+1} \supseteq \cdots \supseteq \mathcal{R}
$$

and

$$
\bigcap_{k=1}^{\infty} \Upsilon_{k}=\mathcal{R} .
$$

Moreover, $\Psi_{k}$ and $\Upsilon_{k}$ are dual to each other (cf. 23, 25, 28,).

As shown above, the hierarchy of $\Upsilon_{k}$ provides the outer approximations of $\mathcal{R}$ and converges monotonically and asymptotically to $\mathcal{R}$. So, $\Upsilon_{k}$ can approximate the completely positive cone $\mathcal{R}$ arbitrarily well. 


\section{Linear optimization With the CP CONE AND NORM CONE}

In this section, we formulate the CP-matrix approximation problem (1.2) as a linear optimization problem with the cone of moments and the $p$-norm cone. The duality is also discussed.

Introducing a variable $\gamma \in \mathbb{R}_{+}$, we transform (1.2) to the following problem:

$$
\begin{array}{cl}
\min _{X, \gamma} & \gamma \\
\text { s.t. } & \|X-C\|_{p} \leq \gamma, \\
& A_{i} \bullet X=b_{i}, i=1, \ldots, m_{e}, \\
& A_{i} \bullet X \geq b_{i}, i=m_{e}+1, \ldots, m, \\
& X \in \mathcal{C} \mathcal{P}_{n} .
\end{array}
$$

Let $Y=X-C$. Then (3.1) can be equivalently written as

$$
\begin{aligned}
\min _{X, Y, \gamma} & \gamma \\
\text { s.t. } & A_{i} \bullet X=b_{i}, i=1, \ldots, m_{e}, \\
& A_{i} \bullet X \geq b_{i}, i=m_{e}+1, \ldots, m, \\
& X-Y=C, \\
& X \in \mathcal{C} \mathcal{P}_{n}, \\
& (Y, \gamma) \in \mathcal{K}_{p} .
\end{aligned}
$$

The Lagrange function of (3.2) is:

$$
\begin{aligned}
L(X, Y, \gamma, \lambda, P, S, Z, \xi)= & \gamma-\sum_{i=1}^{m_{e}} \lambda_{i}\left(A_{i} \bullet X-b_{i}\right)-\sum_{i=m_{e}+1}^{m} \lambda_{i}\left(A_{i} \bullet X-b_{i}\right) \\
& -(X-Y-C) \bullet P-X \bullet S-Y \bullet Z-\gamma \xi
\end{aligned}
$$

Denote by $\mathcal{F}(3.2)$ the feasible set of $(3.2)$. Then, the Lagrange dual problem of (3.2) is

$$
\begin{aligned}
& \max _{\lambda, P, S, Z, \xi} \inf _{(X, Y, \gamma) \in \mathcal{F} \text { (3.2) }} L(X, Y, \gamma, \lambda, P, S, Z, \xi) \\
& \text { s.t. } \quad \lambda_{i} \geq 0, i=m_{e}+1, \ldots m \text {, } \\
& P \in \mathcal{S}_{n} \text {, } \\
& S \in \mathcal{C O P}{ }_{n}, \\
& (Z, \xi) \in \mathcal{K}_{p}^{*} \text {. }
\end{aligned}
$$

Let $b=\left(b_{1}, \ldots, b_{m}\right)^{T}$. Then (3.4) can be simplified as:

$$
\begin{array}{cl}
\max _{\lambda, S, Z} & b^{T} \lambda+C \bullet Z \\
\text { s.t. } & \sum_{i=1}^{m} \lambda_{i} A_{i}+S+Z=0, \\
& \lambda_{i} \geq 0, i=m_{e}+1, \ldots, m, \\
& (S,(Z, 1)) \in \mathcal{C O P} \mathcal{P}_{n} \times \mathcal{K}_{p}^{*} .
\end{array}
$$

Denote

$$
\begin{aligned}
x & =\operatorname{vech}(X) \in \mathbb{R}^{\bar{n}} \quad \text { with } \quad \bar{n}=n(n+1) / 2, \\
a_{i} & =\operatorname{vech}\left(2 E_{n}-I_{n}\right) \circ \operatorname{vech}\left(A_{i}\right) \in \mathbb{R}^{\bar{n}}, i=1, \ldots, m,
\end{aligned}
$$


where "о" denotes the Hadamard product, $E_{n}$ is the all-ones matrix and $I_{n}$ the identity matrix of order $n$ respectively, Then, (3.2) can be formulated as the following linear optimization problem:

$$
\begin{aligned}
\vartheta_{P}=\min _{x, Y, \gamma} & \gamma \\
\text { s.t. } & a_{i}^{T} x=b_{i}, i=1, \ldots, m_{e} \\
& a_{i}^{T} x \geq b_{i}, i=m_{e}+1, \ldots, m, \\
& x-\operatorname{vech}(Y)=\operatorname{vech}(C), \\
& (x,(Y, \gamma)) \in \mathcal{R} \times \mathcal{K}_{p},
\end{aligned}
$$

where $\mathcal{R}$ is given by (2.6). The dual problem of $(P)$ is

$$
\begin{aligned}
& (D): \quad \text { s.t. } \quad \sum_{i=1}^{m} \lambda_{i} a_{i}+s+\operatorname{vech}\left(2 E_{n}-I_{n}\right) \circ \operatorname{vech}(Z)=0 \text {, } \\
& \lambda_{i} \geq 0, i=m_{e}+1, \ldots, m, \\
& (s,(Z, 1)) \in \mathcal{P} \times \mathcal{K}_{p}^{*},
\end{aligned}
$$

where $\mathcal{P}$ is given by (2.8).

By weak duality, for all feasible points $(x, Y, \gamma)$ in $(P)$ and $(\lambda, s, Z)$ in $(D)$, we have

$$
\vartheta_{P} \geq \vartheta_{D}
$$

The theorem below shows when the strong duality holds, i.e, the equality holds for (3.6).

Theorem 3.1. For problems $(P)$ and $(D)$,

(i) If there exist $\left(x^{*}, Y^{*}, \gamma^{*}\right) \in \mathcal{F}(P)$ and $\left(\lambda^{*}, s^{*}, Z^{*}\right) \in \mathcal{F}(D)$ such that $\gamma^{*}-$ $b^{T} \lambda^{*}-C \bullet Z^{*}=0$, then $\left(x^{*}, Y^{*}, \gamma^{*}\right)$ and $\left(\lambda^{*}, s^{*}, Z^{*}\right)$ are the minimizers of $(P)$ and $(D)$, respectively.

(ii) If there exists $(x, Y, \gamma)$ such that $(x, Y, \gamma) \in \mathcal{F}(P), a_{i} \bullet x>b_{i}\left(i=m_{e}+\right.$ $1, \ldots, m)$ and $(x,(Y, \gamma)) \in \operatorname{int}\left(\mathcal{R} \times \mathcal{K}_{p}\right)$, then we have $\vartheta_{P}=\vartheta_{D}$. Furthermore, if $\vartheta_{P}$ is finite, then there exists an optimal $\left(\lambda^{*}, s^{*}, Z^{*}\right)$ such that $\vartheta_{P}=b^{T} \lambda^{*}+C \bullet Z^{*}=\vartheta_{D}$. If $\left(x^{*}, Y^{*}, \gamma^{*}\right)$ is the minimizer of $(P)$, then there exists a dual feasible point $(\bar{\lambda}, \bar{s}, \bar{Z})$ such that $\gamma^{*}-b^{T} \bar{\lambda}-C \bullet \bar{Z}=0$.

(iii) If there exists $(\lambda, s, Z) \in \mathcal{F}(D)$ such that $\lambda_{i}>0\left(i=m_{e}+1, \ldots, m\right)$ and $(s,(Z, 1)) \in \operatorname{int}\left(\mathcal{P} \times \mathcal{K}_{p}^{*}\right)$, then we have $\vartheta_{P}=\vartheta_{D}$. Furthermore, if $\vartheta_{D}$ is finite, then there exists an optimal $\left(x^{*}, Y^{*}, \gamma^{*}\right)$ such that $\vartheta_{P}=\gamma^{*}=\vartheta_{D}$. If $\left(\lambda^{*}, s^{*}, Z^{*}\right)$ is the minimizer of $(D)$, then there exists a primal feasible $(\bar{x}, \bar{Y}, \bar{\gamma})$ such that $\bar{\gamma}-b^{T} \lambda^{*}-C \bullet Z^{*}=0$.

Theorem 3.1 can be implied by the standard strong duality theory (cf. [1]), so we omit the proof here. For convenience, if there exists $(\lambda, s, Z) \in \mathcal{F}(\mathrm{D})$ such that $\lambda_{i}>0\left(i=m_{e}+1, \ldots, m\right)$ and $(s,(Z, 1)) \in \operatorname{int}\left(\mathcal{P} \times \mathcal{K}_{p}^{*}\right)$, we call $(D)$ have a relative interior.

\section{A Semidefinite Algorithm}

In this section, we present a semidefinite algorithm for the CP-matrix approximation problem and study its convergence properties. 
4.1. A semidefinite algorithm. As shown in (2.18) and (2.22), $\mathcal{R}$ and $\mathcal{P}$ have nice relaxations $\Upsilon_{k}$ and $\Psi_{k}$, respectively. By (2.20) and (2.21), the $k$-th order relaxation of $(P)$ can be defined as

$$
\begin{aligned}
\vartheta_{P}^{k}=\min _{x, Y, \gamma, \tilde{x}} & \gamma \\
\text { s.t. } \quad & a_{i}^{T} x=b_{i}, i=1, \ldots, m_{e}, \\
& a_{i}^{T} x \geq b_{i}, i=m_{e}+1, \ldots, m, \\
& x-\operatorname{vech}(Y)=\operatorname{vech}(C), \\
& x=\left.\tilde{x}\right|_{E}, \\
& (\tilde{x},(Y, \gamma)) \in \Gamma_{k} \times \mathcal{K}_{p},
\end{aligned}
$$

and the dual problem of $\left(P^{k}\right)$ is

$$
\begin{aligned}
\vartheta_{D}^{k}=\max _{\lambda, s, Z} & b^{T} \lambda+C \bullet Z \\
\text { s.t. } \quad & \sum_{i=1}^{m} \lambda_{i} a_{i}+s+\operatorname{vech}\left(2 E_{n}-I_{n}\right) \circ \operatorname{vech}(Z)=0, \\
& \lambda_{i} \geq 0, i=m_{e}+1, \ldots, m, \\
& (s,(Z, 1)) \in \Psi_{k} \times \mathcal{K}_{p}^{*} .
\end{aligned}
$$

Both $\left(P^{k}\right)$ and $\left(D^{k}\right)$ are SDP problems, so they can be solved efficiently.

Clearly, $\vartheta_{P}^{k} \leq \vartheta_{P}$ and $\vartheta_{D}^{k} \leq \vartheta_{D}$ for all $k$. Suppose $\left(x^{*, k}, Y^{*, k}, \gamma^{*, k}, \tilde{x}^{*, k}\right)$ is a minimizer of $\left(P^{k}\right)$ and $\left(\lambda^{*, k}, s^{*, k}, Z^{*, k}\right)$ is a maximizer of $\left(D^{k}\right)$. If $x^{*, k}=\left.\tilde{x}^{*, k}\right|_{E} \in \mathcal{R}$, then $\vartheta_{P}^{k}=\vartheta_{P}$ and $\left(x^{*, k}, Y^{*, k}, \gamma^{*, k}\right)$ is a minimizer of $(P)$, i.e., the relaxation $\left(P^{k}\right)$ is exact for solving $(P)$. In this case, if $\vartheta_{P}^{k}=\vartheta_{D}^{k}$, then $\vartheta_{D}^{k}=\vartheta_{D}$ and $\left(\lambda^{*, k}, s^{*, k}, Z^{*, k}\right)$ is a maximizer of $(D)$. If the relaxation $\left(P^{k}\right)$ is infeasible, then $(P)$ is infeasible, i.e., (1.2) is infeasible.

Based on the above, we propose a semidefinite algorithm for the CP-matrix approximation problem (1.2).

Algorithm 4.1 (A semidefinite algorithm for the CP-matrix approximation problem).

Step 0. Input $C \in \mathcal{S}_{n}$ and $\Delta$ as (2.3). Let $k:=2$.

Step 1. Solve the primal-dual pair $\left(P^{k}\right)-\left(D^{k}\right)$. If $\left(P^{k}\right)$ is infeasible, stop and output that $(P)$ is infeasible; otherwise, compute an optimal solution $\left(x^{*, k}, Y^{*, k}, \gamma^{*, k}, \tilde{x}^{*, k}\right)$ of $\left(P^{k}\right)$. Let $t:=1$. 4 .

Step 2. Let $\hat{x}:=\left.\tilde{x}^{*, k}\right|_{2 t}$. If the rank condition (2.13) is not satisfied, go to Step

Step 3. Compute the finitely atomic measure $\mu$ admitted by $\hat{x}$ :

$$
\mu=\rho_{1} \delta\left(u_{1}\right)+\cdots+\rho_{r} \delta\left(u_{r}\right),
$$

where $\rho_{i}>0, u_{i} \in \Delta, r=\operatorname{rank}\left(M_{t}(\hat{x})\right)$ and $\delta\left(u_{i}\right)$ is the Dirac measure supported on the point $u_{i} \in \Delta$.

Step 4. If $t<k$, set $t:=t+1$ and go to Step 2; otherwise, set $k:=k+1$ and go to Step 1.

If (1.2) is feasible, Algorithm 4.1 can give a projection matrix of a symmetric matrix onto the intersection of a set of linear constraints and the $\mathrm{CP}$ cone. A CPdecomposition of the projection matrix can also be obtained. If (1.2) is infeasible, Algorithm 4.1 can give a certificate for the infeasibility.

We use Step 2 to check whether $\left.\tilde{x}^{*, k}\right|_{2 t}$ is flat or not. Nie [28] showed it might be possible that $x^{*, k} \in \mathcal{R}$ while $\left.\tilde{x}^{*, k}\right|_{2 t}$ is not flat for all $t$. In such cases, we can apply the algorithms given in [27, 36] to check whether $x^{*, k} \in \mathcal{R}$ or not. 
We use Henrion and Lasserre's method in [19] to get a $r$-atomic $\Delta$-measure for $\hat{x}$, which can further produce the $\mathrm{CP}$-decomposition of the projection matrix.

We discuss how to solve $\left(P^{k}\right)$ for different $p$-norm cone in section 4.3.

4.2. Convergence properties. We give the asymptotic convergence of Algorithm 4.1 as follows.

Theorem 4.2. Let $E$ and $\Delta$ be as in (2.2) and (2.11), respectively. Suppose (P) is feasible and $(D)$ has a relative interior point. Algorithm 4.1 has the following properties:

(i) For all $k$ sufficiently large, $\left(D^{k}\right)$ has a relative interior point and $\left(P^{k}\right)$ has a minimizer $\left(x^{*, k}, Y^{*, k}, \gamma^{*, k}, \tilde{x}^{*, k}\right)$.

(ii) The sequence $\left\{\left(x^{*, k}, Y^{*, k}, \gamma^{*, k}\right)\right\}$ is bounded, and each of its accumulation points is a minimizer of $(P)$. The sequence $\left\{\gamma^{*, k}\right\}$ converges to the minimum of (1.2).

Proof. (i) Let $\left(\lambda^{0}, s^{0}, Z^{0}\right) \in \mathcal{F}(\mathrm{D})$ with $\lambda_{i}^{0}>0\left(i=m_{e}+1, \ldots, m\right)$, and $\left(s^{0},\left(Z^{0}, 1\right)\right) \in$ $\operatorname{int}\left(\mathcal{P} \times \mathcal{K}_{p}^{*}\right)$. Then, $\left.s^{0}\right|_{\Delta}>0$ (cf. [28, Lemma 3.1]). Note that since $\Delta$ is compact, there exist $\epsilon>0$ and $\delta>0$ such that

$$
\left.s\right|_{\Delta}-\epsilon>\epsilon, \quad \forall s \in B\left(s^{0}, \delta\right) .
$$

By [30, Theorem 6], there exists $N_{0}>0$ such that

$$
s-\epsilon \in I_{2 N_{0}}(h)+Q_{N_{0}}(g), \quad \forall s \in B\left(s^{0}, \delta\right) .
$$

So $\left(D^{k}\right)$ has a relative interior point for all $k \geq N_{0}$, thus the strong duality holds for $\left(P^{k}\right)$ and $\left(D^{k}\right)$. As $(P)$ is feasible, the relaxation problem $\left(P^{k}\right)$ is also feasible. So, $\left(P^{k}\right)$ has a minimizer $\left(x^{*, k}, Y^{*, k}, \gamma^{*, k}, \tilde{x}^{*, k}\right)$ (cf. 11, Theorem 2.4.I]).

(ii) We first show $\left\{\left(x^{*, k}, Y^{*, k}, \gamma^{*, k}\right)\right\}$ is bounded. Let $\left(\lambda^{0}, s^{0}, Z^{0}\right)$ and $\epsilon$ be as in the proof of (i). The set $I_{2 N_{0}}(h)+Q_{N_{0}}(g)$ is dual to $\Gamma_{N_{0}}$. For all $k \geq N_{0}$, we have $\tilde{x}^{*, k} \in \Gamma_{N_{0}}$ and

$$
\begin{gathered}
0 \leq\left\langle s^{0}-\epsilon, \tilde{x}^{*, k}\right\rangle=\left\langle s^{0}, \tilde{x}^{*, k}\right\rangle-\epsilon\left\langle 1, \tilde{x}^{*, k}\right\rangle, \\
\left\langle\left(s^{0}, Z^{0}, 1\right),\left(x^{*, k}, Y^{*, k}, \gamma^{*, k}\right)\right\rangle=\gamma^{*, k}-b^{T} \lambda^{0}-C \bullet Z^{0} .
\end{gathered}
$$

Since $\gamma^{*, k} \leq \vartheta_{P}$ and $\left\langle s^{0}, \tilde{x}^{*, k}\right\rangle=\left\langle s^{0}, x^{*, k}\right\rangle \leq\left\langle\left(s^{0}, Z^{0}, 1\right),\left(x^{*, k}, Y^{*, k}, \gamma^{*, k}\right)\right\rangle$, it holds that

We get

$$
\left\langle s^{0}, \tilde{x}^{*, k}\right\rangle \leq T_{0}:=\vartheta_{P}-b^{T} \lambda^{0}-C \bullet Z^{0} .
$$

$$
\begin{gathered}
0 \leq\left\langle s^{0}-\epsilon, \tilde{x}^{*, k}\right\rangle \leq T_{0}-\epsilon\left(\tilde{x}^{*, k}\right)_{\mathbf{0}}, \\
\left(\tilde{x}^{*, k}\right)_{\mathbf{0}} \leq T_{1}:=T_{0} / \epsilon .
\end{gathered}
$$

Note that $I(h)+Q(g)$ is archimedean, following the line of proof given in 28 , Theorem 4.3 (ii)], we can obtain that the sequence $\left\{x^{*, k}\right\}$ is bounded. Due to the relationships between the definitions of $x, Y$ and $\gamma$, we know $\left\{\left(x^{*, k}, Y^{*, k}, \gamma^{*, k}\right)\right\}$ is bounded.

Suppose $\left(x^{*}, Y^{*}, \gamma^{*}\right)$ is an accumulation point of $\left\{\left(x^{*, k}, Y^{*, k}, \gamma^{*, k}\right)\right\}$. Without loss of generality, we assume

$$
\left(x^{*, k}, Y^{*, k}, \gamma^{*, k}\right) \rightarrow\left(x^{*}, Y^{*}, \gamma^{*}\right), \quad k \rightarrow \infty .
$$

Since $x^{*, k} \in \Upsilon_{k}$, by (2.22) and (2.23), we have $x^{*} \in \bigcap_{k=1}^{\infty} \Upsilon_{k}=\mathcal{R}$. Note that $\left(x^{*, k}, Y^{*, k}, \gamma^{*, k}\right) \in \mathcal{F}\left(P^{k}\right)$, we further obtain $\left(x^{*}, Y^{*}, \gamma^{*}\right) \in \mathcal{F}(P)$. Hence,

$$
\vartheta_{P} \leq \gamma^{*} \text {. }
$$


Since $\left(P^{k}\right)$ is a relaxation problem of $(P)$ and $\left(x^{*, k}, Y^{*, k}, \gamma^{*, k}\right)$ is a minimizer of $\left(P^{k}\right)$, we have

$$
\vartheta_{P} \geq \gamma^{*, k}, k=1,2, \ldots
$$

Taking $k \rightarrow \infty$, we get

$$
\vartheta_{P} \geq \lim _{k \rightarrow \infty} \gamma^{*, k}=\gamma^{*}
$$

which together with (4.1) implies that

$$
\vartheta_{P}=\gamma^{*} \text {. }
$$

So, $\left(x^{*}, Y^{*}, \gamma^{*}\right)$ is a minimizer of $(P)$, and the sequence $\left\{\gamma^{*, k}\right\}$ converges to the minimum of $(P)$.

Remark 4.3. If (1.2) is feasible, then, under some general conditions [29, 31, which is almost necessary and sufficient, we can get a flat extension $\tilde{x}^{*, k}$ by solving the hierarchy of $\left(P^{k}\right)$, within finitely many steps [28, Section 4].

4.3. Subproblem solving. We discuss how to solve the subproblem $\left(P^{k}\right)$ in Algorithm 4.1 for different $p$-norm cone $\mathcal{K}_{p}(p=1,2, \infty, F)$.

1. 1-norm or $\infty$-norm cone. The 1-norm and $\infty$-norm are the same for symmetric matrices. Let $Y=Y^{+}-Y^{-}$, where $Y^{+}, Y^{-} \geq 0$ and $Y^{+}, Y^{-} \in \mathcal{S}_{n}$. Then $\left(P^{k}\right)$ can be transformed to the following problem:

$$
\begin{array}{cl}
\min _{\mathbf{x}, Y^{+}, Y^{-}, \gamma, \tilde{x}} & \gamma \\
\text { s.t. } & a_{i}^{T} x=b_{i}, i=1, \ldots, m_{e}, \\
& a_{i}^{T} x \geq b_{i}, i=m_{e}+1, \ldots, m, \\
& x-\operatorname{vech}\left(Y^{+}-Y^{-}\right)=\operatorname{vech}(C), \\
& \tilde{E}_{j} \bullet\left(Y^{+}+Y^{-}\right) \leq \gamma, j=1, \ldots, n, \\
& Y^{+}, Y^{-} \geq 0, Y^{+}, Y^{-} \in \mathcal{S}_{n}, \\
& x=\left.\tilde{x}\right|_{E}, \tilde{x} \in \Gamma_{k},
\end{array}
$$

where $\tilde{E}_{j}$ is the matrix whose $j$-th column is of all ones and other entries are zeros.

(4.3) is a linear optimization problem with linear matrix inequalities. It can be solved by the softwares GloptiPoly 3 [20] and SeDuMi 35].

2. 2-norm cone. Note that $(Y, \gamma) \in \mathcal{K}_{2}$ if and only if $\left(\begin{array}{cc}\gamma I_{n} & Y \\ Y^{T} & \gamma I_{n}\end{array}\right) \succeq 0$. Since $Y=X-C$, we can transform $\left(P^{k}\right)$ to the following problem:

$$
\begin{aligned}
& \min _{x, \gamma, \tilde{x}} \gamma \\
& \text { s.t. } \quad a_{i}^{T} x=b_{i}, i=1, \ldots, m_{e} \text {, } \\
& a_{i}^{T} x \geq b_{i}, i=m_{e}+1, \ldots, m \text {, } \\
& \left(\begin{array}{cc}
\gamma I_{n} & \operatorname{vech}^{-1}(x)-C \\
\left(\operatorname{vech}^{-1}(x)-C\right)^{T} & \gamma I_{n}
\end{array}\right) \succeq 0, \\
& x=\left.\tilde{x}\right|_{E}, \tilde{x} \in \Gamma_{k},
\end{aligned}
$$

where $\operatorname{vech}^{-1}(\cdot)$ denotes the inverse of the linear operator vech $(\cdot)$.

(4.4) can also be solved by the softwares GloptiPoly 3 [20] and SeDuMi [35].

3. F-norm cone. Let

$$
y=\operatorname{vech}\left(\sqrt{2} E_{n}+(1-\sqrt{2}) I_{n}\right) \circ \operatorname{vech}(Y) .
$$


Then $(Y, \gamma) \in \mathcal{K}_{F}$ if and only if $(y, \gamma) \in \mathcal{L}_{\bar{n}+1}$, where

$$
\mathcal{L}_{\bar{n}+1}=\left\{(y, \gamma) \in \mathbb{R}^{\bar{n}+1}:\|y\|_{2} \leq \gamma\right\}
$$

is the second-order cone (or Lorentz cone). Since $Y=X-C,\left(P^{k}\right)$ can be transformed to the following problem:

$$
\begin{aligned}
\min _{x, y, \gamma, \tilde{x}} & \gamma \\
\text { s.t. } & a_{i}^{T} x=b_{i}, i=1, \ldots, m_{e}, \\
& a_{i}^{T} x \geq b_{i}, i=m_{e}+1, \ldots, m, \\
& y=\operatorname{vech}\left(\sqrt{2} E_{n}+(1-\sqrt{2}) I_{n}\right) \circ(x-\operatorname{vech}(C)), \\
& x=\left.\tilde{x}\right|_{E}, \\
& (\tilde{x},(y, \gamma)) \in \Gamma_{k} \times \mathcal{L}_{\bar{n}+1} .
\end{aligned}
$$

(4.5) is a linear optimization problem with the second-order cone and linear matrix inequalities. It can be solved by the softwares GloptiPoly $3[20$ and SeDuMi [35].

\section{Numerical experiments}

In this section, we present some numerical experiments for computing the projection of a matrix onto the intersection of a set of linear constraints and the $\mathrm{CP}$ cone by using Algorithm 4.1. A CP-decomposition of the projection matrix is also given if the problem is feasible. The experiments are implemented on a laptop with an Intel Core i5-2520M CPU and 4GB of RAM, using Matlab R2012b. We only display 4 digits for each number.

\subsection{CP-approximation in 1-norm or $\infty$-norm.}

Example 5.1. Consider the symmetric matrix $C$ given as:

$$
C=\left(\begin{array}{llll}
2 & 1 & 1 & 1 \\
1 & 2 & 2 & 1 \\
1 & 2 & 6 & 5 \\
1 & 1 & 5 & 6
\end{array}\right)
$$

It can be checked that $C$ is double nonnegative. Since a symmetric double nonnegative matrix with the order less than or equal to 4 is $\mathrm{CP}$ (cf. [2]), we have $C \in \mathcal{C P}_{4}$.

Case 1. Consider (1.2) without linear constraints, i.e., we compute the projection of $C$ onto $\mathcal{C P}_{4}$ in 1-norm.

Algorithm 4.1 terminates at $k=3$, with $\gamma^{*, k}=0.0000$ and $x^{*, k} \in \mathcal{R}$. So, $X^{*}=$ $C$. This verifies that $C$ is CP. The CP-decomposition of $C$ is $C=\sum_{i=1}^{5} \rho_{i} u_{i} u_{i}^{T}$, where the points and their weights are:

$$
\begin{array}{ll}
\rho_{1}=3.0297, & u_{1}=(0.0000,0.6287,0.6491,0.4284)^{T}, \\
\rho_{2}=7.6746, & u_{2}=(0.0000,0.0000,0.6347,0.7728)^{T}, \\
\rho_{3}=2.6969, & u_{3}=(0.4767,0.3641,0.7779,0.1875)^{T}, \\
\rho_{4}=1.0808, & u_{4}=(0.7669,0.6418,0.0000,0.0000)^{T}, \\
\rho_{5}=1.5179, & u_{5}=(0.7036,0.0000,0.0000,0.7106)^{T} .
\end{array}
$$


Case 2. Consider (1.2) with the $\mathrm{CP}$ cone and the linear constraints $A_{i} \bullet X=$ $b_{i}(i=1,2)$, where

$$
\begin{aligned}
& A_{1}=I_{4}, \quad A_{2}=\left(\begin{array}{llll}
0 & 1 & 0 & 1 \\
1 & 0 & 1 & 0 \\
0 & 1 & 0 & 1 \\
1 & 0 & 1 & 0
\end{array}\right), \\
& b_{1}=10, \quad b_{2}=12 .
\end{aligned}
$$

Algorithm 4.1 terminates at $k=3$ with $\gamma^{*, k}=3.0209$ and $x^{*, k} \in \mathcal{R}$. The optimal solution is

$$
X^{*}=\left(\begin{array}{cccc}
0.2709 & 0.1572 & 0.9582 & 0.5928 \\
0.1572 & 0.6302 & 1.1918 & 1.0000 \\
0.9582 & 1.1918 & 4.7709 & 4.0582 \\
0.5928 & 1.0000 & 4.0582 & 4.3280
\end{array}\right)
$$

The CP-decomposition of $X^{*}$ is $X^{*}=\sum_{i=1}^{3} \rho_{i} u_{i} u_{i}^{T}$, where the points and their weights are:

$$
\begin{array}{ll}
\rho_{1}=0.8735, & u_{1}=(0.2014,0.6294,0.7506,0.0000)^{T}, \\
\rho_{2}=3.1791, & u_{2}=(0.2677,0.0000,0.8209,0.5044)^{T}, \\
\rho_{3}=5.9473, & u_{3}=(0.0357,0.2186,0.5993,0.7693)^{T} .
\end{array}
$$

Case 3. Consider (1.2) with the $\mathrm{CP}$ cone and the linear constraints $A_{i} \bullet X=$ $b_{i}(i=1,2)$, where

$$
\begin{aligned}
& A_{1}=\left(\begin{array}{cccc}
1 & -1 & 1 & -1 \\
-1 & 2 & -2 & 2 \\
1 & -2 & 3 & -3 \\
-1 & 2 & -3 & 4
\end{array}\right), \quad A_{2}=-I_{4} \\
& b_{1}=5, \quad b_{2}=-19 .
\end{aligned}
$$

Algorithm 4.1 terminates at $k=2$ as $\left(P^{k}\right)$ is infeasible. So, (1.2) is infeasible.

Case 4. Consider (1.2) with the $\mathrm{CP}$ cone and the linear constraints $A_{1} \bullet X=b_{1}$ and $A_{2} \bullet X \geq b_{2}$, where $A_{i}, b_{i}(i=1,2)$ are the same as in Case 3 .

Algorithm 4.1 terminates at $k=3$ with $\gamma^{*, k}=1.6916$. The optimal solution is

$$
X^{*}=\left(\begin{array}{cccc}
0.8232 & 0.8946 & 1.4094 & 1.0000 \\
0.8946 & 0.9745 & 1.4394 & 1.0000 \\
1.4094 & 1.4394 & 5.9043 & 4.9991 \\
1.0000 & 1.0000 & 4.9991 & 4.3094
\end{array}\right) \text {. }
$$

The CP-decomposition of $X^{*}$ is $X^{*}=\sum_{i=1}^{2} \rho_{i} u_{i} u_{i}^{T}$, where the points and their weights are:

$$
\begin{aligned}
& \rho_{1}=2.0184, \quad u_{1}=(0.5817,0.6464,0.4532,0.1957)^{T}, \\
& \rho_{2}=9.9929, \quad u_{2}=(0.1184,0.1145,0.7412,0.6508)^{T} .
\end{aligned}
$$




\subsection{CP-approximation in 2-norm.}

Example 5.2. Consider the symmetric matrix $C$ given as (cf. [34]):

$$
C=\left(\begin{array}{lllll}
2 & 1 & 1 & 1 & 2 \\
1 & 2 & 2 & 1 & 1 \\
1 & 2 & 6 & 5 & 1 \\
1 & 1 & 5 & 6 & 2 \\
2 & 1 & 1 & 2 & 3
\end{array}\right)
$$

It is shown in 34] that $C \in \mathcal{C P}_{5}$ and the CP-rank of $C$ is 5 .

Case 1. Consider (1.2) with the $\mathrm{CP}$ cone and the linear constraints $A_{i} \bullet X=$ $b_{i}(i=1,2,3)$, where

$$
\begin{aligned}
& A_{1}=I_{5}, \quad A_{2}=\left(\begin{array}{ccccc}
1 & -1 & 1 & -1 & 1 \\
-1 & 2 & -2 & 2 & -2 \\
1 & -2 & 3 & -3 & 3 \\
-1 & 2 & -3 & 4 & -4 \\
1 & -2 & 3 & -4 & 5
\end{array}\right), \quad A_{3}=\left(\begin{array}{lllll}
0 & 1 & 0 & 1 & 0 \\
1 & 0 & 1 & 0 & 1 \\
0 & 1 & 0 & 1 & 0 \\
1 & 0 & 1 & 0 & 1 \\
0 & 1 & 0 & 1 & 0
\end{array}\right), \\
& b_{1}=19, \quad b_{2}=17, \quad b_{3}=24 .
\end{aligned}
$$

Algorithm 4.1 terminates at $k=3$, with $\gamma^{*, k}=0.0000$ and $x^{*, k} \in \mathcal{R}$. So, $X^{*}=C$. This implies that $C$ is not only CP but also satisfies the linear constraints. The CP-decomposition of $C$ is $C=\sum_{i=1}^{5} \rho_{i} u_{i} u_{i}^{T}$, where the points and their weights are:

$$
\begin{array}{ll}
\rho_{1}=5.5421, & u_{1}=(0.0862,0.0000,0.3963,0.7926,0.4553)^{T}, \\
\rho_{2}=3.8751, & u_{2}=(0.6826,0.2828,0.0000,0.0000,0.6738)^{T}, \\
\rho_{3}=7.2866, & u_{3}=(0.1450,0.2334,0.7608,0.5879,0.0000)^{T}, \\
\rho_{4}=0.8380, & u_{4}=(0.0000,0.9438,0.0000,0.0000,0.3306)^{T}, \\
\rho_{5}=1.4582, & u_{5}=(0.0058,0.6122,0.7907,0.0000,0.0000)^{T} .
\end{array}
$$

We obtained a minimal CP-decomposition of $C$. It is different from the minimal $\mathrm{CP}$-decomposition given in [34.

Case 2. Consider (1.2) with the $\mathrm{CP}$ cone and the linear constraints $A_{i} \bullet X=$ $b_{i}(i=1,2,3)$, where $A_{1}, A_{2}, A_{3}, b_{1}, b_{3}$ are the same as in Case 1 , and

$$
b_{2}=50 \text {. }
$$

Algorithm 4.1 terminates at $k=3$ with $\gamma^{*, k}=2.8436$. The optimal solution is

$$
X^{*}=\left(\begin{array}{lllll}
1.6135 & 1.3913 & 2.3928 & 1.6291 & 2.3642 \\
1.3913 & 2.4173 & 2.1301 & 3.1909 & 1.6217 \\
2.3928 & 2.1301 & 5.0301 & 4.1451 & 2.8557 \\
1.6291 & 3.1909 & 4.1451 & 6.0656 & 1.0827 \\
2.3642 & 1.6217 & 2.8557 & 1.0827 & 3.8735
\end{array}\right)
$$

The CP-decomposition of $X^{*}$ is $X^{*}=\sum_{i=1}^{3} \rho_{i} u_{i} u_{i}^{T}$, where the points and their weights are:

$$
\begin{aligned}
& \rho_{1}=6.4943, \quad u_{1}=(0.4251,0.1801,0.4791,0.0000,0.7465)^{T} \\
& \rho_{2}=3.9517, \quad u_{2}=(0.1403,0.0000,0.7829,0.6061,0.0000)^{T} \\
& \rho_{3}=8.5539, \quad u_{3}=(0.2058,0.5079,0.3613,0.7344,0.1723)^{T} .
\end{aligned}
$$


Case 3. Consider (1.2) with the $\mathrm{CP}$ cone and the linear constraints $A_{i} \bullet X=$ $b_{i}(i=1,2,3)$, where $A_{1}, A_{2}, A_{3}, b_{1}, b_{3}$ are the same as in Case 1 , but

$$
b_{2}=-50 \text {. }
$$

Algorithm 4.1 terminates at $k=2$ as $\left(P^{k}\right)$ is infeasible. So, (1.2) is infeasible.

Case 4. Consider (1.2) with the $\mathrm{CP}$ cone and the linear constraints $A_{i} \bullet X=$ $b_{i}(i=1,2)$ and $A_{3} \bullet X \geq b_{3}$, where $A_{i}(i=1,2,3)$ are the same as in Case 1 , and

$$
b_{1}=10, \quad b_{2}=12, \quad b_{3}=-2 .
$$

Algorithm 4.1 terminates at $k=3$, with $\gamma^{*, k}=3.3763$ and $x^{*, k} \in \mathcal{R}$. The optimal solution is:

$$
X^{*}=\left(\begin{array}{lllll}
0.4943 & 0.3541 & 1.2119 & 0.9809 & 0.7703 \\
0.3541 & 0.4565 & 1.3018 & 1.3352 & 0.4720 \\
1.2119 & 1.3018 & 3.8986 & 3.7575 & 1.7180 \\
0.9809 & 1.3352 & 3.7575 & 3.9192 & 1.2798 \\
0.7703 & 0.4720 & 1.7180 & 1.2798 & 1.2316
\end{array}\right) \text {. }
$$

The CP-decomposition of $X^{*}$ is $X^{*}=\sum_{i=1}^{2} \rho_{i} u_{i} u_{i}^{T}$, where the points and their weights are:

$$
\begin{aligned}
& \rho_{1}=1.3602, \quad u_{1}=(0.4277,0.0342,0.4667,0.0000,0.7734)^{T}, \\
& \rho_{2}=8.6396, \quad u_{2}=(0.1686,0.2295,0.6457,0.6735,0.2199)^{T} .
\end{aligned}
$$

\subsection{CP-approximation in $F$-norm.}

Example 5.3. Consider the symmetric matrix $C$ given in Example 5.2

Case 1. Consider (1.2) with the CP cone and the linear constraints $A_{i} \bullet X=$ $b_{i}(i=1,2,3)$, where $A_{i}, b_{i}(i=1,2,3)$ are the same as in Case 1 of Example [5.2.

Algorithm 4.1 terminates at $k=3$, with $\gamma^{*, k}=0.0000$ and $x^{*, k} \in \mathcal{R}$. So, $C \in \mathcal{C P}_{5}$. We get the same CP-decomposition of $C$ as that in Case 1 of Example 5.2 .

Case 2. Consider (1.2) with the $\mathrm{CP}$ cone and the linear constraints $A_{i} \bullet X=$ $b_{i}(i=1,2,3)$, where $A_{i}, b_{i}(i=1,2,3)$ are the same as in Case 2 of Example 5.2.

Algorithm 4.1 terminates at $k=3$ with $\gamma^{*, k}=4.7642$. The optimal solution is

$$
X^{*}=\left(\begin{array}{ccccc}
1.4456 & 1.2748 & 2.0122 & 1.7636 & 2.1333 \\
1.2748 & 1.3345 & 2.1511 & 2.4889 & 1.4697 \\
2.0122 & 2.1511 & 5.5864 & 4.4398 & 3.0769 \\
1.7636 & 2.4889 & 4.4398 & 6.3423 & 0.9011 \\
2.1333 & 1.4697 & 3.0769 & 0.9011 & 4.2912
\end{array}\right) .
$$

The CP-decomposition of $X^{*}$ is $X^{*}=\sum_{i=1}^{4} \rho_{i} u_{i} u_{i}^{T}$, where the points and their weights are:

$$
\begin{array}{ll}
\rho_{1}=2.5727, & u_{1}=(0.5085,0.3250,0.0000,0.0000,0.7973)^{T}, \\
\rho_{2}=7.8537, & u_{2}=(0.1698,0.2829,0.4990,0.8013,0.0000)^{T}, \\
\rho_{3}=4.5924, & u_{3}=(0.1992,0.1020,0.7125,0.0000,0.6650)^{T}, \\
\rho_{4}=3.9812, & u_{4}=(0.3055,0.3115,0.5712,0.5713,0.3962)^{T} .
\end{array}
$$

Case 3. Consider (1.2) with the $\mathrm{CP}$ cone and the linear constraints $A_{i} \bullet X=$ $b_{i}(i=1,2,3)$, where $A_{i}, b_{i}(i=1,2,3)$ are the same as in Case 3 of Example 5.2 . 
JINYAN FAN AND ANWA ZHOU

Algorithm 4.1 terminates at $k=2$ as $\left(P^{k}\right)$ is infeasible. So, (1.2) is infeasible.

Case 4. Consider (1.2) with the CP cone and the linear constraints $A_{i} \bullet X=$ $b_{i}(i=1,2)$ and $A_{3} \bullet X \geq b_{3}$, where $A_{i}(i=1,2,3)$ are the same as in Case 4 of Example 5.2

Algorithm 4.1 terminates at $k=3$, with $\gamma^{*, k}=5.1904$ and $x^{*, k} \in \mathcal{R}$. The optimal solution is:

$$
X^{*}=\left(\begin{array}{ccccc}
0.6441 & 0.3961 & 1.1295 & 0.8842 & 0.9479 \\
0.3961 & 0.4305 & 1.2678 & 1.2371 & 0.5508 \\
1.1295 & 1.2678 & 3.7388 & 3.6771 & 1.5635 \\
0.8842 & 1.2371 & 3.6771 & 3.7860 & 1.1818 \\
0.9479 & 0.5508 & 1.5635 & 1.1818 & 1.4007
\end{array}\right)
$$

The CP-decomposition of $X^{*}$ is $X^{*}=\sum_{i=1}^{2} \rho_{i} u_{i} u_{i}^{T}$, where the points and their weights are:

$$
\begin{aligned}
& \rho_{1}=1.6630, \quad u_{1}=(0.5129,0.1257,0.3173,0.0000,0.7877)^{T}, \\
& \rho_{2}=8.3370, \quad u_{2}=(0.1574,0.2202,0.6545,0.6739,0.2104)^{T} .
\end{aligned}
$$

Example 5.4. Consider the symmetric matrix

$$
C=\left(\begin{array}{llllll}
4 & 5 & 4 & 6 & 4 & 2 \\
5 & 1 & 4 & 7 & 4 & 6 \\
4 & 4 & 4 & 2 & 5 & 4 \\
6 & 7 & 2 & 0 & 3 & 7 \\
4 & 4 & 5 & 3 & 1 & 6 \\
2 & 6 & 4 & 7 & 6 & 4
\end{array}\right)
$$

which is generated randomly in Matlab.

Case 1. Consider (1.2) without linear constraints. Algorithm 4.1 terminates at $k=3$, with $\gamma^{*, k}=9.7852$. So, $C$ is not CP. The projection matrix of $C$ onto $\mathcal{C} \mathcal{P}_{5}$ is

$$
X^{*}=\left(\begin{array}{cccccc}
5.3184 & 4.4216 & 3.6259 & 4.2906 & 3.4447 & 3.6739 \\
4.4216 & 4.8771 & 3.6321 & 4.7517 & 3.9731 & 5.1854 \\
3.6259 & 3.6321 & 4.7970 & 3.0763 & 3.9514 & 3.9240 \\
4.2906 & 4.7517 & 3.0763 & 4.7343 & 3.6374 & 4.9919 \\
3.4447 & 3.9731 & 3.9514 & 3.6374 & 3.7868 & 4.5142 \\
3.6739 & 5.1854 & 3.9240 & 4.9919 & 4.5142 & 6.3710
\end{array}\right) .
$$

The CP-decomposition of $X^{*}$ is $X^{*}=\sum_{i=1}^{4} \rho_{i} u_{i} u_{i}^{T}$, where the points and their weights are:

$$
\begin{array}{cc}
\rho_{1}=7.0443, & u_{1}=(0.3599,0.4862,0.0000,0.5526,0.2300,0.5252)^{T} \\
\rho_{2}=4.6526, & u_{2}=(0.0000,0.2673,0.6042,0.1571,0.4868,0.5494)^{T} \\
\rho_{3}=13.2067, & u_{3}=(0.3707,0.4278,0.3766,0.4028,0.3828,0.4785)^{T} \\
\rho_{4}=4.9810, & u_{4}=(0.7213,0.3047,0.4961,0.2554,0.2749,0.0000)^{T} .
\end{array}
$$


Case 2. Consider (1.2) with the $\mathrm{CP}$ cone and the linear constraints $A_{i} \bullet X=$ $b_{i}(i=1,2)$, where $A_{i}, b_{i}(i=1,2)$ are generated randomly:

$$
\begin{aligned}
A_{1} & =\left(\begin{array}{cccccc}
-12 & 0 & 7 & -5 & 4 & -2 \\
0 & 3 & 1 & -2 & -6 & -13 \\
7 & 1 & 4 & 1 & -9 & 6 \\
-5 & -2 & 1 & 7 & -9 & 10 \\
4 & -6 & -9 & -9 & -19 & 1 \\
-2 & -13 & 6 & 10 & 1 & 13
\end{array}\right), \\
A_{2} & =\left(\begin{array}{cccccc}
-4 & 3 & 11 & 11 & 2 & -5 \\
3 & 6 & 3 & -3 & 5 & -9 \\
11 & 3 & 5 & 0 & -3 & -9 \\
11 & -3 & 0 & 14 & -4 & -16 \\
2 & 5 & -3 & -4 & 7 & -14 \\
-5 & -9 & -9 & -16 & -14 & 3
\end{array}\right), \\
b_{1} & =-17, \quad b_{2}=6 .
\end{aligned}
$$

Algorithm 4.1 terminates at $k=3$, with $y_{1}^{*, k}=11.4970$. The optimal solution is

$$
X^{*}=\left(\begin{array}{cccccc}
5.5277 & 4.9260 & 5.0372 & 4.5381 & 3.1904 & 2.7187 \\
4.9260 & 4.7114 & 4.2671 & 4.8028 & 3.0364 & 3.5882 \\
5.0372 & 4.2671 & 6.0026 & 3.3516 & 3.4975 & 3.0055 \\
4.5381 & 4.8028 & 3.3516 & 5.5691 & 2.9252 & 4.7051 \\
3.1904 & 3.0364 & 3.4975 & 2.9252 & 2.3729 & 3.0340 \\
2.7187 & 3.5882 & 3.0055 & 4.7051 & 3.0340 & 6.9676
\end{array}\right) .
$$

The CP-decomposition of $X^{*}$ is $X^{*}=\sum_{i=1}^{3} \rho_{i} u_{i} u_{i}^{T}$, where the points and their weights are:

$$
\begin{gathered}
\rho_{1}=5.0609, \quad u_{1}=(0.5069,0.5243,0.0000,0.6726,0.0996,0.0766)^{T}, \\
\rho_{2}=7.4131, \quad u_{2}=(0.0000,0.1967,0.0000,0.4359,0.1960,0.8561)^{T}, \\
\rho_{3}=18.6772, \quad u_{3}=(0.4757,0.4030,0.5669,0.3165,0.3303,0.2839)^{T} .
\end{gathered}
$$

Case 3. Consider (1.2) with the CP cone and the linear constraints $A_{i} \bullet X=$ $b_{i}(i=1,2)$, where $A_{i}, b_{i}(i=1,2)$ are generated randomly:

$$
\begin{aligned}
A_{1} & =\left(\begin{array}{cccccc}
8 & -2 & 5 & 6 & 5 & -4 \\
-2 & 10 & 8 & 12 & 17 & 4 \\
5 & 8 & 7 & 6 & -2 & -3 \\
6 & 12 & 6 & 4 & 12 & 7 \\
5 & 17 & -2 & 12 & 10 & -8 \\
-4 & 4 & -3 & 7 & -8 & 9
\end{array}\right), \\
A_{2} & =\left(\begin{array}{cccccc}
-2 & -16 & -12 & 4 & 1 & -5 \\
-16 & 3 & 8 & -3 & -10 & 0 \\
-12 & 8 & -13 & -1 & 11 & 3 \\
4 & -3 & -1 & -3 & 5 & 9 \\
1 & -10 & 11 & 5 & 10 & 3 \\
-5 & 0 & 3 & 9 & 3 & -15
\end{array}\right), \\
b_{1} & =-6, \quad b_{2}=4 .
\end{aligned}
$$

Algorithm 4.1 terminates at $k=2$ as $\left(P^{k}\right)$ is infeasible. So, (1.2) is infeasible. 
Case 4. Consider (1.2) with the $\mathrm{CP}$ cone and the linear constraints $A_{1} \bullet X=$ $b_{1}, A_{2} \bullet X \geq b_{2}$, where $A_{i}, b_{i}(i=1,2)$ are generated randomly:

$$
\begin{aligned}
A_{1} & =\left(\begin{array}{cccccc}
5 & 7 & -4 & -9 & 4 & 9 \\
7 & -2 & 6 & -4 & 7 & -6 \\
-4 & 6 & -17 & -9 & -1 & 6 \\
-9 & -4 & -9 & 5 & -13 & 6 \\
4 & 7 & -1 & -13 & -3 & 1 \\
9 & -6 & 6 & 6 & 1 & -6
\end{array}\right), \\
A_{2} & =\left(\begin{array}{cccccc}
2 & -4 & 6 & 4 & 7 & 1 \\
-4 & -2 & 11 & 2 & 6 & 7 \\
6 & 11 & 12 & -9 & -2 & 7 \\
4 & 2 & -9 & -3 & 0 & 10 \\
7 & 6 & -2 & 0 & 4 & -11 \\
1 & 7 & 7 & 10 & -11 & 11
\end{array}\right), \\
b_{1} & =7, \quad b_{2}=-10 .
\end{aligned}
$$

Algorithm 4.1 terminates at $k=3$, with $\gamma^{*, k}=10.4410$. The optimal solution is

$$
X^{*}=\left(\begin{array}{cccccc}
5.5853 & 4.9676 & 3.4690 & 3.9792 & 3.9314 & 4.6082 \\
4.9676 & 5.0242 & 3.6848 & 4.2784 & 4.1044 & 5.3069 \\
3.4690 & 3.6848 & 3.6883 & 2.3311 & 3.7530 & 3.9202 \\
3.9792 & 4.2784 & 2.3311 & 4.5442 & 2.8846 & 4.8841 \\
3.9314 & 4.1044 & 3.7530 & 2.8846 & 3.9128 & 4.3521 \\
4.6082 & 5.3069 & 3.9202 & 4.8841 & 4.3521 & 6.2313
\end{array}\right) .
$$

The CP-decomposition of $X^{*}$ is $X^{*}=\sum_{i=1}^{3} \rho_{i} u_{i} u_{i}^{T}$, where the points and their weights are:

$$
\begin{array}{cc}
\rho_{1}=3.4136, & u_{1}=(0.7633,0.3769,0.3647,0.0000,0.3772,0.0000)^{T}, \\
\rho_{2}=7.4149, & u_{2}=(0.4695,0.4255,0.0000,0.6215,0.1124,0.4467)^{T}, \\
\rho_{3}=18.1576, & u_{3}=(0.3287,0.4196,0.4221,0.3042,0.4285,0.5115)^{T} .
\end{array}
$$

Example 5.5. Consider the computing time of projecting a random symmetric matrix onto the $\mathrm{CP}$ cone. For each $n=2,3, \ldots, 10$, we generate 50 random symmetric $n \times n$ matrices.

Table 1 shows the average time (seconds) consumed by Algorithm4.1 to compute the projection matrix onto the $\mathrm{CP}$ cone.

\begin{tabular}{cccccccccc}
\hline $\mathrm{n}$ & 2 & 3 & 4 & 5 & 6 & 7 & 8 & 9 & 10 \\
\hline Time & 0.36 & 0.53 & 0.80 & 1.56 & 4.76 & 21.37 & 101.29 & 428.21 & 1732.21 \\
\hline
\end{tabular}

TABLE 1. The average time for computing the CP projection matrix.

\section{Conclusions}

We study the CP-matrix approximation problem of projecting a symmetric matrix onto the intersection of a set of linear constraints and the CP cone. It includes the feasibility problem and the CP projection problem as special cases. We formulate the problem as the linear optimization with the cone of moments and the 
$p$-norm cone $(p=1,2, \infty$, or $F)$. A semidefinite algorithm (i.e., Algorithm 4.1) is presented for it. Its convergence is also studied. If the problem is infeasible, we can get a certificate for it. If the problem is feasible, we can get a projection matrix; moreover, a CP-decomposition of the projection matrix can also be obtained. Numerical results show that Algorithm 4.1 is efficient in solving the CP-matrix approximation problem.

\section{REFERENCES}

[1] A. Ben-Tal and A. Nemirovski, Lectures on Modern Convex Optimization: Analysis, Algorithms, and Engineering Applications, MPS-SIAM Series on Optimization, SIAM, Philadelphia, 2001.

[2] A. Berman and N. Shaked-Monderer, Completely Positive Matrices, World Scientific, 2003.

[3] I. M. Bomze And E. DE KLeRK, Solving standard quadratic optimization problems via linear, semidefinite and copositive programming, J. Global Optim., 24 (2002), pp. 163-185.

[4] I. M. Bomze, Copositive optimization-recent developments and applications, Eur. J. Oper. Res., 216 (2012), pp. 509-520.

[5] Stephen Boyd and Lieven Vandenberghe, Convex optimization, Cambridge University Press, Cambridge, 2004.

[6] S. BuRER, On the copositive representation of binary and continuous nonconvex quadratic programs, Math. Program., Ser. A, 120 (2009), pp. 479-495.

[7] S. Burer, Copositive programming, Handbook on semidefinite, conic and polynomial optimization, 201-C218, Internat. Ser. Oper. Res. Management Sci., 166, Springer, New York, 2012.

[8] J.Q. Chen And S. Burer, Globally solving nonconvex quadratic programming problems via completely positive programming, Math. Program. Comput., 4 (2012), pp. 33-52.

[9] F. Cucker, J. Peña And V. Roshchina, Round-off estimates for second-order conic feasibility problems, C. R. Math. Acad. Sci. Paris, 350 (2012), pp. 639-C641.

[10] F. CUCKer AND J. PEÑA, A primal-dual algorithm for solving polyhedral conic systems with a finite-precision machine, SIAM J. Optim., 12 (2001/02), pp. 522-C554.

[11] R. Curto and L. Fialkow, Truncated $K$-moment problems in several variables, J. Operator Theory, 54 (2005), pp. 189-226.

[12] E. De Klerk and D. V. Pasechnik, Approximation of the stability number of a graph via copositive programming, SIAM J. Optim., 12 (2002), pp. 875-892.

[13] P. J. Dickinson and L. Gijben, On the computational complexity of membership problems for the completely positive cone and its dual, Computational Optimization and Applications, 57 (2014), pp. 403-415.

[14] H. Dong And K. Anstreicher, Separating doubly nonnegative and completely positive matrices, Math. Program., Ser. A, 137 (2013), pp. 131-153.

[15] M. Dür, Copositive Programming-a Survey, in M. Diehl, F. Glineur, E. Jarlebring and W. Michiels, Eds., Recent Advances in Optimization and its Applications in Engineering, Springer, 2010, pp. 3-20.

[16] L. Fialkow And J. NiE, The truncated moment problem via homogenization and flat extensions, J. Funct. Anal., 263 (2012), pp. 1682-1700.

[17] M. Hall JR, Combinatorial theory, Blaisdell Publishing Co., Boston, 1967.

[18] J. W. Helton AND J. NIE, A semidefinite approach for truncated K-moment problems, Found. Comput. Math., 12 (2012), pp. 851-881.

[19] D. Henrion and J. B. Lasserre, Detecting global optimality and extracting solutions in GloptiPoly, Positive polynomials in control, Lecture Notes in Control and Inform. Sci. Springer, Berlin, 312 (2005), pp. 293-310.

[20] D. Henrion, J. B. Lasserre and J. Loefberg, GloptiPoly 3: moments, optimization and semidefinite programming, Optim. Methods Softw., 24 (2009), pp. 761-779.

[21] J. B. LASSERRE, Global optimization with polynomials and the problem of moments, SIAM J. Optim., 11(2001), pp. 796-817.

[22] J. B. LASSERRE, A semidefinite programming approach to the generalized problem of moments, Math. Program., 112 (2008), pp. 65-92. 
[23] J. B. Lasserre, Moments, Positive Polynomials and Their Applications, Imperial College Press, 2009.

[24] J. B. LASSERRe, New approximations for the cone of copositive matrices and its dual, Math. Program., Ser. A, 144 (2014), pp. 265-276.

[25] M. LAURENT, Sums of squares, moment matrices and optimization over polynomials, Emerging Applications of Algebraic Geometry, Vol. 149 of IMA Volumes in Mathematics and its Applications, M. Putinar and S. Sullivant (eds), Springer, 2009, pp. 157-270.

[26] K. G. Murty And S. N. Kabadi, Some NP-complete problems in quadratic and nonlinear programming. Math. Program., 39 (1987), pp. 117-129.

[27] J. NIE, The $A$-truncated K-moment problem, Found. Comput. Math., DOI 10.1007/s10208014-9225-9.

[28] J. NIE, Linear optimization with cones of moments and nonnegative polynomials, Math. Program., Ser. B, DOI 10.1007/s10107-014-0797-6.

[29] J. NiE, Optimality conditions and finite convergence of Lasserres hierarchy, Math. Program., Ser. A, 146 (2014), pp. 97-121.

[30] J. Nie And M. Schweighofer, On the complexity of putinars positivstellensatz, J. Complexity, 23 (2007), pp. 135-150.

[31] J. Nie And K. RANESTAD, Algebraic degree of polynomial optimization, SIAM J. Optim., 20 (2009), pp. 485-502.

[32] J. Peña, J. Vera And L. Zuluaga, Computing the stability number of a graph via linear and semidenite programming, SIAM J. Optim., 18 (2007), pp. 87-105.

[33] M. Putinar, Positive polynomials on compact semi-algebraic sets, Ind. Aniv. Math. J., 42 (1993), pp. 969-984.

[34] J. Sponsel And M. Dür, Factorization and cutting planes for completely positive matrices by copositive projection, Math. Program., Ser. A, 143 (2014), pp. 211-229.

[35] J. F. Sturm, SeDuMi 1.02: A MATLAB toolbox for optimization over symmetric cones, Optim. Methods Softw., 11 \& 12 (1999), pp. 625-653.

[36] A. Zhou And J. Fan, The CP-matrix completion problem, SIAM. J. Matrix Anal. Appl., 45 (2014), pp. 127-142.

[37] A. ZhOU AND J. FAN, Interiors of completely positive cones, submitted.

Department of Mathematics, and MOE-LSC, Shanghai Jiao Tong University, ShangHAI 200240, P.R. CHINA

E-mail address: jyfan@sjtu.edu.cn

Department of Mathematics, Shanghai Jiao Tong University, Shanghai 200240, P.R. CHINA

E-mail address: congcongyan@sjtu.edu.cn 